\title{
Effects of Postprandial Lipemia on Plasma Cholesterol Metabolism
}

\author{
Graciela R. Castro and Christopher J. Fielding \\ Cardiovascular Research Institute and Department of Physiology, University of California, San Francisco, California 94143
}

\begin{abstract}
Cholesterol net transport, esterification, and cholesteryl ester transfer have been determined in plasma during fasting, and postprandially, after a high fat-cholesterol meal. Significant rises in plasma triglyceride, phospholipid, and free cholesterol were associated with increases in cholesterol net transport, esterification, and transfer (all $P<0.005$ ), which were well correlated in individual subjects $(r>0.60)$. Essentially, the whole of free cholesterol required for such increased esterification was derived from cell membranes, when cultured fibroblasts were present, despite the increased level of free cholesterol in postprandial plasma; most of the additional cholesteryl ester generated was transferred to the low and very low density lipoproteins (LDL and VLDL) of plasma. Postprandial LDL (the major carrier of free and ester cholesterol and phospholipids among the acceptor lipoproteins) contained significantly decreased ratios of free cholesterol to phospholipid $(P<0.001)$, which may modulate the increased transfer of cholesteryl ester to VLDL and LDL. These data suggest that the presence of postprandial acceptor lipoproteins in plasma may play an important role in stimulating the "reverse" transport of cholesterol from peripheral cells for hepatic degradation, which is effective even after the ingestion of dietary cholesterol.
\end{abstract}

\section{Introduction}

In human plasma, the free and ester cholesterol composition of the circulating lipoproteins is determined in large part by the activity of three coupled reactions. These are the transport of free cholesterol for esterification from either cell membranes or plasma lipoproteins; the esterification of cholesterol of either origin by the action of lecithin:cholesterol acyltransferase (LCAT) ${ }^{1}$; and the transfer of the cholesteryl esters so formed to acceptor lipoproteins. In normal plasma, a major part of LCAT-derived cholesteryl esters is transferred to the low and very low density lipoproteins (LDL and VLDL) (1). Welldefined abnormalities of this reaction sequence have been found in several genetic and acquired hyperlipidemias that are associated with an increased risk of atherosclerotic vascular

G. R. Castro is a visiting scientist from La Universidad del Litoral, Santa Fe, Argentina.

Received for publication \& May 1984 and in revised form 21 November 1984

1. Abbreviations used in this paper: apo E, apolipoprotein E; BMI, body mass index; CE, cholesteryl ester; FC, free cholesterol; HDL, high density lipoproteins; LCAT, lecithin:cholesterol acyltransferase; LDL, low density lipoproteins; PL, phospholipid; TG, triglyceride; VLDL, very low density lipoproteins.

J. Clin. Invest.

(c) The American Society for Clinical Investigation, Inc. 0021-9738/85/03/874/09 $\$ 1.00$

Volume 75, March 1985, 874-882 disease (2-4). In all of these the pattern is the same. There is a defect in cholesterol net transport from cell membranes to plasma, whose effect is that free cholesterol for esterification is then derived in large part from the plasma lipoproteins. There is also a defect in cholesteryl ester transfer such that in these groups of patients, cholesteryl esters derived from LCAT are transferred not to VLDL and LDL as in normal plasma (even though these lipoproteins are present in a generally increased concentration) but to high density lipoprotein (HDL). In at least one such hyperlipidemia (that associated with Type II or noninsulin-dependent diabetes) it has been shown recently that the block to transfer is mediated via the abnormally high level of free cholesterol present in the VLDL and LDL (4). It seems likely that similar mechanisms may be involved in others at increased risk of atherosclerotic vascular disease (5).

In this study we have measured the effects of the postprandial hyperlipidemia that follows a high fat-cholesterol meal on the rates of cholesterol transport, esterification, and transfer in normal human plasma.

\section{Methods}

The subjects in this study were ten healthy nonobese volunteers (five men and five women) with fasting plasma triglyceride and total cholesterol levels of $103 \pm 36$ and $179 \pm 24 \mathrm{mg} \mathrm{dl}^{-1}$, respectively. Body mass index $\left(B M I=\right.$ weight $/(\text { height })^{2}$ as kilograms $\left.\times 10^{4} / \mathrm{cm}^{2}\right)$ was 21.1 \pm 1.8 . After an overnight fast, a blood sample was collected into one twentieth volume of $0.2 \mathrm{M}$ sodium citrate solution, cooled in ice water, and plasma was obtained by centrifugation $(2,000 \mathrm{~g}, 30 \mathrm{~min}$, $4^{\circ} \mathrm{C}$ ). Each subject then consumed within $10 \mathrm{~min}$ a meal of bacon and eggs, toast, and ice cream containing $67 \mathrm{~g}$ fat (including $0.6 \mathrm{~g}$ cholesterol), $94 \mathrm{~g}$ carbohydrate, and $40 \mathrm{~g}$ protein (1,150 calories). Subsequent blood samples were taken as above at 3, 5-6, and 8-9 $\mathrm{h}$ postprandially, and plasma was obtained as before for analysis of plasma lipids, apolipoproteins, and metabolic activities, as follows.

Determination of cholesterol net transport from cell membranes to plasma. The mass of cholesterol contributed by cell membranes to plasma in response to a given plasma esterification rate was determined using a standard cultured fibroblast assay previously described (2). Similar results have been obtained with other vascular cells (6). Briefly, normal skin fibroblasts were cultured to near confluence in $6-\mathrm{cm}$ plastic dishes containing $10 \% \mathrm{vol} / \mathrm{vol}$ fetal calf serum in Dulbecco's modified Eagle's medium. Under these conditions the cells contained 8-10 $\mu \mathrm{g}$ cholesterol per dish. In some experiments the cells were prelabeled for $24-48 \mathrm{~h}$ with $1,2-{ }^{3} \mathrm{H}$-cholesterol (New England Nuclear, Boston, MA) to a specific activity of $27-45 \times 10^{3} \mathrm{dpm} / \mu \mathrm{g}$ cholesterol (7). The net transport of cholesterol between cell membranes and plasma was determined in a medium of plasma $(1.2 \% \mathrm{vol} / \mathrm{vol}$ in phosphate buffered saline). The plasma had been depleted of fibrinogen by immunoaffinity chromatography, to prevent coagulation during incubation (7). This medium was incubated in plastic dishes in the presence or absence of cells. Samples of medium were taken before and after incubation of the dishes for $60 \mathrm{~min}$ at $37^{\circ} \mathrm{C}$. The free cholesterol content of initial and incubated medium samples was measured by fluorimetry with cholesterol oxidase (8). Cholesterol net transport was determined as [(decrease in medium-free sterol in the empty dishes) - (decrease in medium-free sterol in the dishes containing cells)] (2). 
In the labeled cultures, the rate of cholesterol efflux was determined from cell cholesterol specific activity, and the rate at which cholesterol radioactivity appeared in the unlabeled plasma medium (2). The rate of cholesterol net transport was determined simultaneously as described above. The rate of influx of unlabeled plasma cholesterol was determined as [efflux - net transport].

Total free cholesterol used for LCAT-mediated esterification was given by the rate of decrease in free cholesterol content during incubation of the plasma medium in the absence of cells. The rate at which free cholesterol was supplied to LCAT by the plasma lipoproteins is the difference between the total free cholesterol supplied to LCAT and the rate of cholesterol net transport; that is, the rate of loss of medium free cholesterol in the dishes containing cells. The efficiency of use of cell-derived and plasma free cholesterol for esterification by LCAT was determined as follows. The mass of cell-derived cholesterol esterified was determined by measuring the radioactivity present in cholesteryl esters after ${ }^{3} \mathrm{H}$-cholesterol-labeled cells were incubated with unlabeled plasma medium. Total medium lipids were extracted with chloroform and methanol and were fractionated on silica gel layers on glass plates developed in hexane-diethyl ether-acetic acid 83:16:1 vol/ vol. The mass of cell-derived cholesteryl ester was obtained by dividing the radioactivity in this fraction by cell cholesterol specific activity. The proportion of cell-derived free cholesterol esterified was obtained by dividing this mass by the total mass of cholesterol transported from cell to medium (i.e., cholesterol net transport). This was compared with the proportion of unlabeled medium free cholesterol esterified under the same conditions, which was determined fluorimetrically (8). The ratio between these two determinations represents the relative efficiency at which cell-derived and medium-derived cholesterol are used for esterification by LCAT in this system, and was $4.5 \pm 1.5$ ( $n$ $=5$ ) under the conditions used in this study.

Determination of cholesterol esterification rates in plasma. Esterification in total plasma was determined as previously shown $(2,8)$, as the rate of decrease in the free cholesterol concentration of plasma incubated in the presence of $10 \mathrm{mM}$ Tris- $\mathrm{HCl}$ and $1 \mathrm{mM}$ sodium EDTA, pH 7.4, at $37^{\circ} \mathrm{C}$. Free cholesterol in pentuplicate initial and incubated samples was determined fluorimetrically using cholesterol oxidase.

Determination of cholesteryl ester transfer. Cholesteryl ester transfer to VLDL and LDL was determined as the difference in the rates of cholesteryl ester accumulation in whole plasma, and in whole plasma from which VLDL and LDL had been precipitated with dextran sulfate and $\mathrm{MgCL}_{2}$ (4). Plasma was diluted threefold with $0.15 \mathrm{M} \mathrm{NaCl}$ and brought to $10 \mathrm{mM}$ Tris- $\mathrm{HCl}, 1 \mathrm{mM}$ sodium EDTA, pH 7.4. Pentuplicate initial $100-\mu \mathrm{l}$ samples of dilute plasma were mixed with $0.9 \mathrm{ml}$ of $0.15 \mathrm{M} \mathrm{NaCl}$, then $1.0 \mathrm{ml}$ of methanol. A further $1-\mathrm{ml}$ portion of diluted plasma was mixed with 0.05 vol of $2 \mathrm{M} \mathrm{MgCl}_{2}$ and the same volume of dextran sulfate solution in distilled water $(20 \mathrm{mg} /$ $\mathrm{ml}$; dextran sulfate sodium salt, Pharmacia Fine Chemicals, Piscataway, $\mathrm{NJ})$. The precipitate of VLDL and LDL was placed in ice for $15 \mathrm{~min}$, then centrifuged $\left(2,000 \mathrm{~g}, 30 \mathrm{~min}, 4^{\circ} \mathrm{C}\right)$. Pentuplicate $100-\mu 1$ portions of the supernatant solution containing high density lipoproteins (HDL) were taken into $0.9 \mathrm{ml} 0.15 \mathrm{M} \mathrm{NaCl}$ and $1 \mathrm{ml}$ of methanol for analysis. The remaining diluted plasma was then incubated $\left(60 \mathrm{~min}, 37^{\circ} \mathrm{C}\right)$, then samples of whole plasma, and the supernatant from $\mathrm{MgCl}_{2}$ dextran sulfate precipitation, were taken as initially. $1 \mathrm{ml}$ of chloroform was added to each aqueous methanol extract, and portions of the chloroform phase were taken for analysis of cholesteryl ester, using cholesterol esterase and oxidase (8). The fraction of cholesteryl ester synthesized by LCAT, which had been incorporated into the HDL fraction of plasma, was determined as the ratio (increase in supernatant cholesteryl ester)/(increase in total plasma cholesteryl ester). The rate of transfer of cholesteryl ester to VLDL and LDL is (total increase in plasma cholesteryl ester) - (increase in HDL cholesteryl ester). The fraction of newly synthesized cholesteryl ester incorporated into VLDL and LDL is given as (rate of transfer of cholesteryl ester to VLDL and LDL)/(total synthesis of cholesteryl ester in plasma).

Fractionation of plasma lipoproteins. Plasma lipoproteins were fractionated on the basis of their affinity for heparin-agarose. Plasma (3-4 ml) was passed through a column $(1.2 \times 40 \mathrm{~cm})$ of heparinagarose (heparin-Sepharose, Pharmacia Fine Chemicals) equilibrated with $0.15 \mathrm{M} \mathrm{NaCl}, 1 \mathrm{mM}$ EDTA, pH 7.4. Fractions containing detectable nonadsorbed protein or lipids were pooled. The adsorbed fraction of plasma proteins was then eluted with $3 \mathrm{M} \mathrm{NaCl}, 1 \mathrm{mM}$ EDTA, then dialysed against $0.15 \mathrm{M} \mathrm{NaCl}, 1 \mathrm{mM}$ EDTA. The nonadsorbed fraction contained the HDL, together with a fraction of VLDL containing no detectable apolipoprotein E (apo E) (4); the adsorbed fraction contained LDL together with a fraction of VLDL containing apo $\mathrm{E}$. These fractions derived from fasting and postprandial plasma were recombined in the proportions present in native plasma, as described earlier for diabetic and normoglycemic plasma (4), and cholesterol net transport and esterification and cholesteryl ester transfer were determined on such mixtures using the methods described above. In some experiments, both the adsorbed and nonadsorbed pools from heparin-agarose were further fractionated by preparative ultracentrifugation. The samples were brought to $d 1.019 \mathrm{~g} / \mathrm{ml}$ by addition of $\mathrm{KBr}$ solution, then centrifuged $\left(35,000 \mathrm{rpm}, 24 \mathrm{~h}, 4^{\circ} \mathrm{C}\right)$ in the 40.3 rotor of a preparative ultracentrifuge (L3-40 or L3-50; Beckman Instruments, Inc., Fullerton, CA). The supernatant and infranatant fractions were analyzed for their lipid content. Free and esterified cholesterol was determined fluorimetrically as described above. Triglyceride was determined by the chromotropic acid method (9). Phospholipid was determined as inorganic phosphate with ammonium molybdate (10). Recovery of lipids through the fractionation procedure was $86-97 \%$, compared with the original plasma lipid mass applied for chromatography. Electrofocussing of fasting and postprandial VLDL was carried out in polyacrylamide gel (11).

Immunoassay of plasma apolipoproteins. Apolipoproteins A-1, A-2, B, C-3, D, and E were assayed in fasting and postprandial plasma with specific radial immunoassays previously described $(3,4,12)$. Pure apoprotein antigens were isolated from normal human plasma by ultracentrifugation, delipidation with ethanol and diethyl ether, and molecular sieve and DEAE-cellulose chromatography in the presence of $6 \mathrm{M}$ urea. Each was free of detectable impurity by basic and sodium dodecyl sulfate polyacrylamide gel electrophoresis. Antibodies were raised to each pure apoprotein in rabbits. Radial immunodiffusion assays were carried out with $5 \% \mathrm{vol} / \mathrm{vol}$ antiserum in $1 \% \mathrm{wt} / \mathrm{vol}$ agarose and $0.05 \mathrm{M}$ barbital buffer, $\mathrm{pH}$ 8.2. The protein content of the antigens used as standard is expressed in terms of the Lowry protein assay (13).

Data analysis. The significance of differences in plasma metabolic parameters between fasting and postprandial samples was determined by paired $t$ test.

\section{Results}

Effects of postprandial lipemia on plasma lipids and apolipoproteins. The uptake of dietary lipids into the plasma was associated with significant increases in the triglyceride, phospholipid, and unesterified cholesterol levels of plasma (Fig. 1). At the 5-h interval, triglyceride had increased from $103 \pm 36$ to $167 \pm 47 \mathrm{mg} \mathrm{dl}^{-1}(P<0.001)$, free cholesterol had increased from $38 \pm 6$ to $40 \pm 7 \mathrm{mg} \mathrm{dl}^{-1}(P<0.005)$, and phospholipid had increased from $190 \pm 31$ to $214 \pm 36 \mathrm{mg} \mathrm{dl}^{-1}(P<0.001)$. However, the change in total plasma cholesterol with fat feeding (from $179 \pm 24$ to $181 \pm 25 \mathrm{mg} \mathrm{dl}^{-1}$ ) did not reach significance. In studies of the time course of postprandial lipid levels, comparable increases were obtained at 3 and $6 \mathrm{~h}$. Apoprotein levels were therefore compared in fasting plasma, and $5 \mathrm{~h}$ postprandially. As shown in Table $\mathrm{I}$, changes in circulating apolipoprotein levels did not reach significance, except for the level of apolipoprotein C-III, a minor component of triglyceride-rich lipoproteins $(P<0.01)$.

Effects on cholesterol net transport. The movement of cholesterol between cell membranes and plasma was assayed 

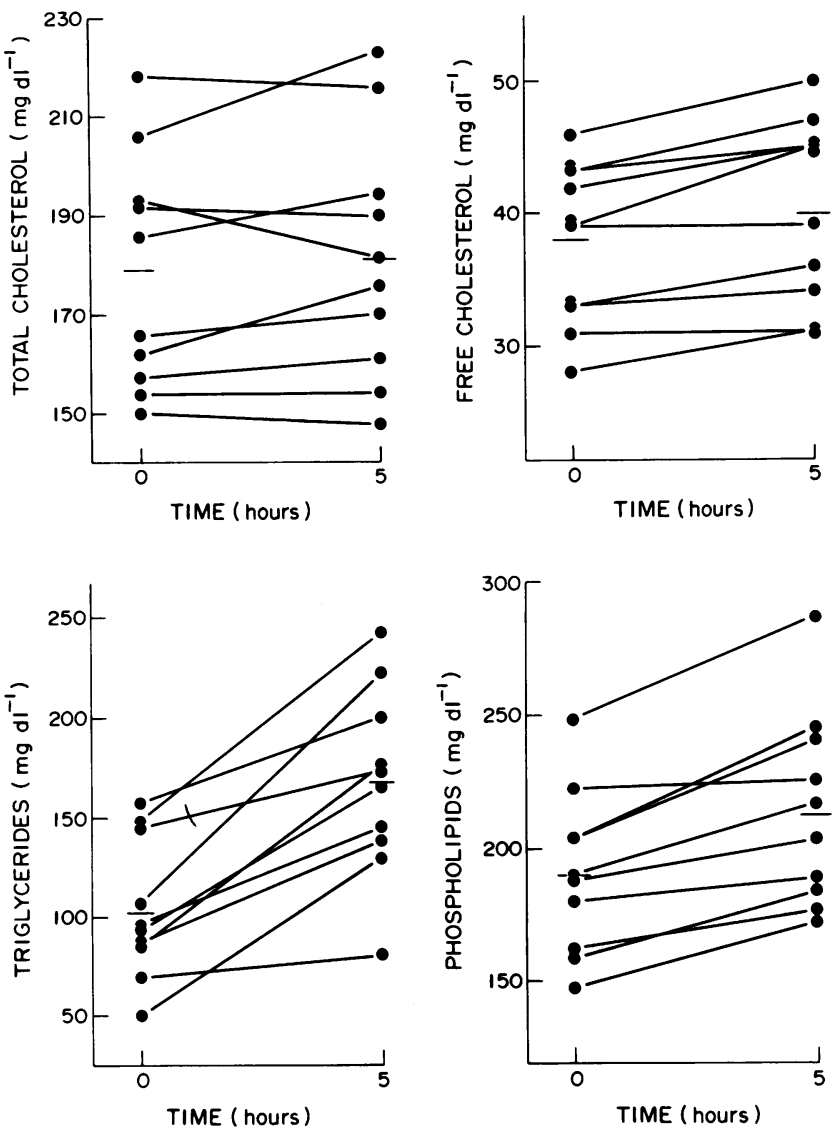

Figure 1. Plasma lipid concentrations in fasting and postprandial plasma. Plasma from blood collected before $(0)$ and $5 \mathrm{~h}$ after the standard high fat-cholesterol meal was extracted with chloroform and methanol, and portions of the total lipid extract were assayed for total cholesterol (top left), free cholesterol (top right), triglyceride (bottom left), and phospholipid (bottom right) (9-11). Total cholesterol was determined as the sum of free and esterified cholesterol. The horizontal bars represent the means of plasma concentrations.

in fasting plasma and $5 \mathrm{~h}$ postprandially (Fig. 2). The mean cholesterol net transport rate per dish of cultured cells between the cell membranes and plasma medium (1.2\% vol/vol) was $0.37 \pm 0.17 \mu \mathrm{g} \mathrm{h}^{-1}$ for fasting plasma and $0.56 \pm 0.17 \mu \mathrm{g} \mathrm{h}^{-1}$ for postprandial plasma $(P<0.005)$, equivalent to a change of $(0.56-0.37) /(0.012 \times 2)$ or $7.9 \mu \mathrm{g}$ free cholesterol $\mathrm{ml}^{-1} \mathrm{~h}^{-1}$ supplied to plasma, under the conditions of these experiments.

Effects of postprandial lipemia on cholesterol esterification. As shown in Fig. 3, postprandial lipemia was also accompanied by a significant rise in the rate of plasma cholesterol esterification (from $17.0 \pm 4.1$ to $26.5 \pm 7.4 \mu \mathrm{g}$ cholesterol esterified $\mathrm{ml}^{-1}$ plasma $\mathrm{h}^{-1}(P<0.001)$. This response continued over the whole time course followed (Fig. 4). The change in mean utilization of HDL free cholesterol for esterification during lipemia was variable between individuals (Fig. 5). While significant for the study group as a whole (mean value, $4.5 \pm 4.3$ $\mu \mathrm{g}$ free cholesterol $\mathrm{ml}^{-1} \mathrm{~h}^{-1}$ for fasting plasma and 7.0 $\pm 2.4 \mu \mathrm{g}$ free cholesterol $\mathrm{ml}^{-1} \mathrm{~h}^{-1}$ for postprandial plasma, $P<0.05$ ), this mean increase $\left(+2.5 \mu \mathrm{g} \mathrm{ml}^{-1}\right)$ was much less than the total increase in plasma esterification rate $(+9.5 \mu \mathrm{g} \mathrm{ml}$ plasma $h^{-1}$, Fig. 3) or than the increase supplied by cell membranes $\left(+7.9 \mu \mathrm{g} \mathrm{ml} \mathrm{m}^{-1}\right.$ plasma $\left.\mathrm{h}^{-1}\right)$. Thus, the overall consequence of the increase in plasma triglyceride-rich lipo- proteins was that increased free cholesterol was required for LCAT activity but this was derived in large part from cell membranes $(\sim 75 \%)$ and in lesser part from HDL, and hardly at all from the VLDL and LDL of the plasma. There was an increase in the efficiency with which cell membrane cholesterol was returned to the plasma compartment, associated with postprandial lipemia following a high fat-cholesterol meal. When esterification was measured with a synthetic liposome substrate under conditions where esterification was independent of plasma lipoprotein concentrations (14), there was no change in $\left(<2 \mu \mathrm{g} \mathrm{ml}^{-1} \mathrm{~h}^{-1}\right)$ fasting and postprandial cholesteryl ester synthesis rates. This indicates that the increase in esterification rate seen in whole plasma represents an activation of an unchanged level of plasma LCAT.

Effects of postprandial lipemia on cholesteryl ester transfer. As shown in Fig. 6, lipemia was also associated with an increase in the rate of transfer of cholesteryl esters to VLDL and LDL (from 8.2 \pm 4.1 to $16.8 \pm 7.6 \mu \mathrm{g}$ cholesterol as cholesteryl ester transferred $\mathrm{ml}^{-1}$ plasma $\mathrm{h}^{-1}, P<0.005$ ). As shown in Fig. 7 , this change in the relative reactivity of VLDL + LDL and of HDL was maintained over the 9-h sampling period. Since the increase in rate of cholesterol esterification in plasma was $9.5 \mu \mathrm{g} \mathrm{ml}^{-1}$ plasma $\mathrm{h}^{-1}$, it is apparent that the increased rate of cholesteryl ester transfer to VLDL and LDL under the same conditions $\left(+8.6 \mu \mathrm{g} \mathrm{ml}^{-1}\right.$ plasma $\left.\mathrm{h}^{-1}\right)$ represents almost the whole of such increased synthesis. In summary, in postprandial lipemia, the major part of free cholesterol required for the increased activity of LCAT is derived from cell membranes, while the major part of the extra cholesteryl ester generated by LCAT is transferred to VLDL and LDL.

Effects of postprandial lipoproteins isolated by heparinagarose. Cholesterol net transport, cholesterol esterification, and cholesteryl ester transfer were determined in experiments in which postprandial VLDL and LDL obtained by heparinagarose were incorporated into fasting plasma, or the same lipoproteins were replaced in the nonadsorbed fraction of postprandial plasma. The replacement of postprandial VLDL and LDL in postprandial plasma resulted in a decrease of $0.27 \pm 0.12 \mu \mathrm{g} \mathrm{h}^{-1}(n=3)$ in free cholesterol transported from cells to medium, which can be compared with a difference of $0.20 \pm 0.17 \mu \mathrm{g}$ between transport rates in intact fasting and postprandial plasma. When postprandial VLDL and LDL were incorporated into fasting plasma, there was an increase of 8.0 $\pm 3.1 \mu \mathrm{g} \mathrm{ml}^{-1}$ plasma $\mathrm{h}^{-1}(n=3)$ in cholesterol esterified relative to the original fasting plasma. This can be compared with the difference of $8.2 \pm 3.7 \mu \mathrm{g} \mathrm{ml}^{-1}$ plasma $\mathrm{h}^{-1}$ between esterification rates in unfractionated fasting and postprandial plasma in the same subjects. Finally, when postprandial VLDL and LDL were incorporated into fasting plasma and cholesteryl ester transfer determined, transfer was increased by $7.0 \pm 2.9$ $\mu \mathrm{g} \mathrm{ml}^{-1}$ plasma $\mathrm{h}^{-1}(n=3)$. This can be compared with the difference of $6.7 \pm 3.3 \mu \mathrm{g} \mathrm{ml}^{-1}$ plasma $\mathrm{h}^{-1}$ between intact fasting and postprandial plasma under the same conditions. These data suggest that the major part or all of the stimulatory effect of postprandial plasma is due to the properties of VLDL and LDL in postprandial plasma.

Correlations between cholesterol transport, esterification, and transfer in postprandial lipemia. It was suggested earlier (1) that the membrane-to-plasma transport of cholesterol, its esterification, and transfer represent coupled reactions, i.e., sequential reactions whose rates are interdependent. When the fasting and postprandial values of cholesterol net transport 


\begin{tabular}{|c|c|c|c|c|c|c|c|c|c|}
\hline & \multirow[b]{2}{*}{ Sex } & \multirow[b]{2}{*}{ Age } & \multirow[b]{2}{*}{ BMI } & \multicolumn{6}{|l|}{ Apolipoproteins } \\
\hline & & & & A-I & A-II & B & C-III & D & $\mathbf{E}$ \\
\hline & & & & $\mu g / m l$ & & & & & \\
\hline \multirow[t]{2}{*}{1} & $\mathbf{M}$ & 29 & 18.6 & 1,215 & 440 & 545 & 50 & 55 & 33 \\
\hline & & & & 1,200 & 445 & 550 & 52 & 62 & 30 \\
\hline \multirow[t]{2}{*}{2} & $F$ & 25 & 20.8 & 1,305 & 460 & 895 & 86 & 70 & 30 \\
\hline & & & & 1,305 & 460 & 870 & 92 & 63 & 30 \\
\hline \multirow[t]{2}{*}{3} & $\mathbf{F}$ & 41 & 24.1 & 1,240 & 465 & 600 & 31 & 49 & 26 \\
\hline & & & & 1,300 & 400 & 620 & 31 & 50 & 27 \\
\hline \multirow[t]{2}{*}{4} & $\mathbf{M}$ & 29 & 20.4 & 1,193 & 346 & 805 & 62 & 72 & 50 \\
\hline & & & & 1,200 & 353 & 805 & 63 & 66 & 53 \\
\hline \multirow[t]{2}{*}{5} & $\mathbf{F}$ & 28 & 18.5 & 1,200 & 335 & 690 & 45 & 71 & 35 \\
\hline & & & & 1,190 & 323 & 680 & 45 & 72 & 35 \\
\hline \multirow[t]{2}{*}{6} & $\mathbf{M}$ & 38 & 23.2 & 1,092 & 383 & 632 & 59 & 45 & 40 \\
\hline & & & & 1,071 & 410 & 656 & 61 & 49 & 39 \\
\hline \multirow[t]{2}{*}{7} & $\mathbf{M}$ & 29 & 20.7 & 1,104 & 375 & 579 & 52 & 62 & 42 \\
\hline & & & & 982 & 383 & 563 & 55 & 62 & 42 \\
\hline \multirow[t]{2}{*}{8} & $F$ & 40 & 22.2 & 1,425 & 320 & 520 & 65 & 67 & 56 \\
\hline & & & & 1,525 & 310 & 578 & 76 & 65 & 56 \\
\hline \multirow[t]{2}{*}{9} & $\mathbf{F}$ & 41 & 21.5 & 1,562 & 435 & 929 & 44 & 44 & 41 \\
\hline & & & & 1,530 & 452 & 931 & 54 & 46 & 43 \\
\hline \multirow[t]{2}{*}{10} & $F$ & 34 & 21.4 & 1,365 & 400 & 595 & 46 & 66 & 52 \\
\hline & & & & 1,378 & 383 & 579 & 53 & 65 & 56 \\
\hline \multirow[t]{2}{*}{ Means } & & $33 \pm 6$ & $21.1 \pm 1.8$ & $1,270 \pm 147$ & $397 \pm 54$ & $678 \pm 146$ & $54 \pm 15$ & $59 \pm 10$ & $40 \pm 10$ \\
\hline & & & & $1,269 \pm 175$ & $391 \pm 52$ & $683 \pm 137$ & $58 \pm 17$ & $60 \pm 9$ & $41 \pm 11$ \\
\hline
\end{tabular}

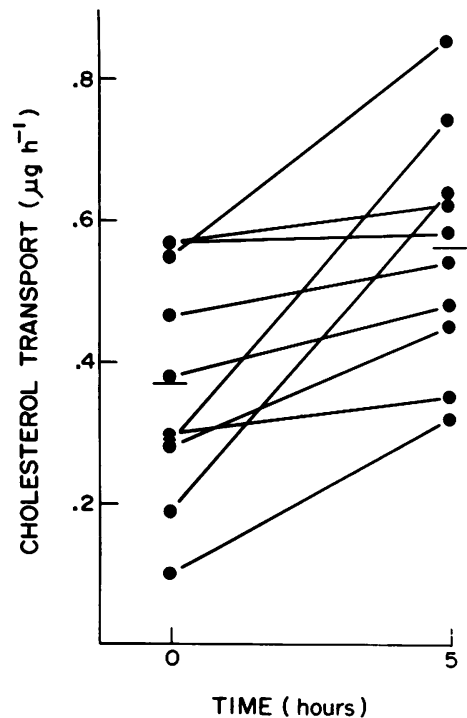
determined as the difference in decrease of free cholesterol in the empty dishes, and the dishes containing cells. Rates refer to transport between cells and medium in the assay conditions described under Methods.

Figure 2. Cholesterol net transport between cultured fibroblasts and medium containing fasting or $5 \mathrm{~h}$ postprandial plasma $(1.2 \%$ $\mathrm{vol} / \mathrm{vol}$ in phosphate-buffered saline) (8). Incubations were of pentuplicate dishes containing cells near confluence, or empty dishes, carried out at $37^{\circ} \mathrm{C} .3 \mathrm{ml}$ of medium was added to each dish, and an initial 1-ml sample was taken for extraction with chloroform and methanol; then the remaining $2 \mathrm{ml}$ was incubated for $60 \mathrm{~min}$. A second milliliter was then taken for extraction, and free cholesterol was determined enzymatically in both initial and incubated samples. Net transport was and cholesterol esterification are compared (Fig. 8), their differences ( $\Delta$ net transport, $\Delta$ esterification) were correlated $(r=0.60$, slope 1.08). Similar results were obtained when

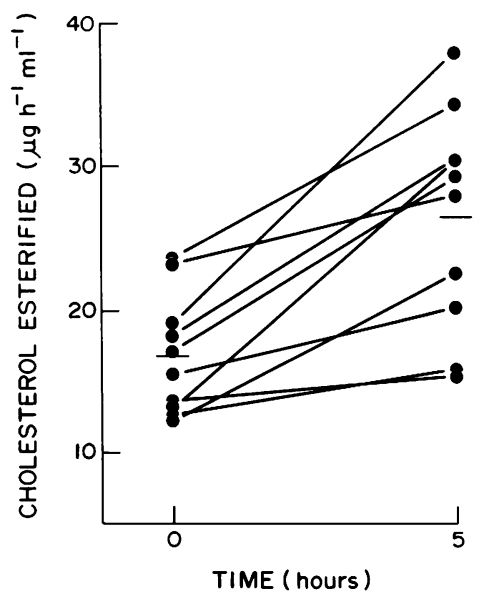

Figure 3. Cholesterol esterification in fasting and in 5 h-postprandial plasma. Pentuplicate samples of plasma were extracted initially, the remaining plasma incubated, and then the same number of samples was taken after $60 \mathrm{~min}$ at $37^{\circ} \mathrm{C}$, for determination of free cholesterol content. Esterification rate is expressed as the rate of decrease in plasma free cholesterol under these conditions (8). This decrease in free cholesterol was inhibited

(>98\%) when the incubation was carried out in the presence of 2 $\mathrm{mM}$ dithiobis (2-nitrobenzoic acid), an inhibitor of LCAT-mediated cholesterol esterification. 


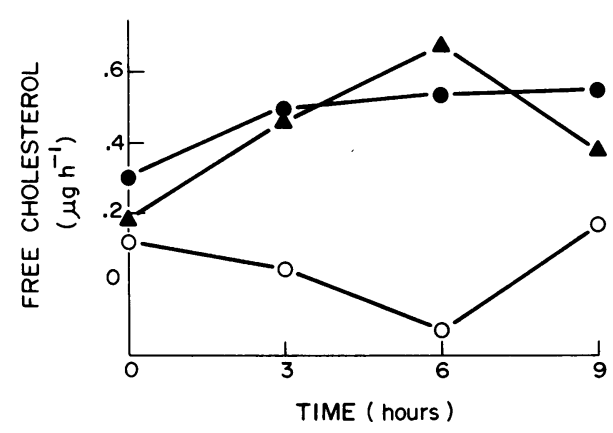

Figure 4. Postprandial time course of plasma cholesterol esterification rate $(-\bullet-)$, cholesterol net transport (cells $\rightarrow$ medium) $(-\Delta-)$, and the rate of esterification of cholesterol from plasma lipoproteins $(-0-)$. Net transport is expressed as in Fig. 2, in terms of the transport of free cholesterol in dishes of fibroblasts during a 60-min incubation at $37^{\circ} \mathrm{C}$, and total cholesterol esterification was determined from the medium of empty dishes incubated with the same plasma medium. Since total esterification rate is the same in the presence or absence of cells (7), the free cholesterol derived from plasma lipoproteins is equivalent to total esterification - net transport in the same units. The data are representative of that obtained in four studies over this time course.

differences ( $\Delta$ esterification, $\Delta$ transfer) in fasting and postprandial esterification and transfer rates were compared (Fig. 9) $(r=0.65$, slope 0.76$)$. Thus, not only were individual values of transport and transfer well correlated with esterification rate, but the increase in rates mediated in the same lipemia was also significantly coupled $(P<0.05)$ and numerically approximately equal.

Mechanism of the modification of cholesterol transport in postprandial lipemia. When the rate of cholesterol efflux from cell membranes was determined using ${ }^{3} \mathrm{H}$-cholesterol-labeled cells, no consistent difference was obtained between efflux rates into fasting and postprandial plasma $(0.55 \pm 0.11$ vs.

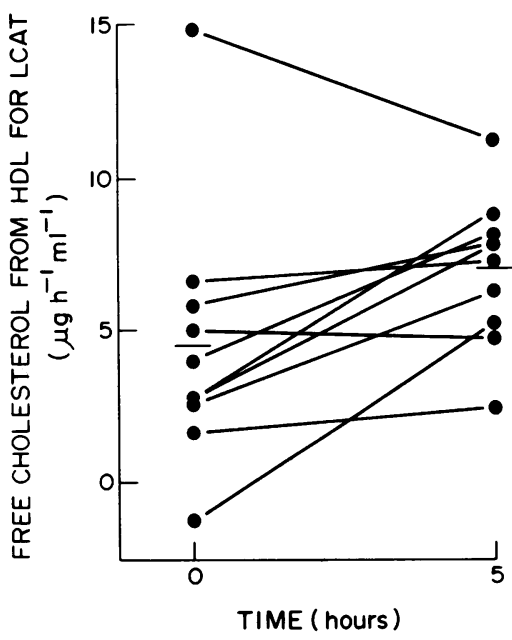

Figure 5. Free cholesterol in HDL used for LCAT-mediated esterification in fasting and $5 \mathrm{~h}$ postprandial plasma. Utilization was determined as the rate of decrease in HDL cholesterol during the esterification of cholesterol in plasma. Initial HDL free cholesterol was determined enzymatically after precipitation of VLDL and LDL with $\mathrm{Mg}_{2+-}$-dextran sulfate. The remaining plasma was then incubated for 60 min at $37^{\circ} \mathrm{C}$, then $\mathrm{HDL}$ cholesterol was determined after precipitation as initially.

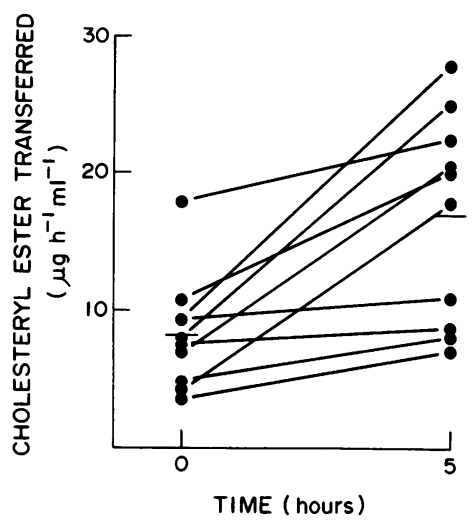

$\left.0.57 \pm 0.15 \mu \mathrm{g} \mathrm{h}^{-1}, P>0.1\right)$. When the rate of influx of cholesterol from the plasma medium to the cells was determined (as efflux - net transport) (Fig. 10), a consistent decrease was found (from $0.29 \pm 0.10 \mu \mathrm{g} \mathrm{h}^{-1}$ in fasting plasma to $0.13 \pm 0.11$ $\mu \mathrm{g} \mathrm{h}^{-1}$ in postprandial plasma) $(P<0.001)$. This difference $\left(0.16 \mu \mathrm{g} \mathrm{h}^{-1}\right)$ is therefore similar to the increase in cholesterol net transport described above (Fig. 2). It indicates that the rate of cholesterol net transport is increased in postprandial plasma primarily because the rate at which free cholesterol returns to the cells from plasma is significantly decreased.

Composition of $V L D L$ and $L D L$ in fasting and postprandial plasma. An earlier study (4) suggested that cholesteryl ester transfer rates were determined by VLDL and LDL lipid composition. The lipid composition of these lipoproteins was determined in fasting and postprandial plasma with lipoproteins fractionated by heparin-agarose chromatography. VLDL ( $d$ $<1.019 \mathrm{~g} \mathrm{ml}^{-1}$ ) was separated by centrifugation from the adsorbed and nonadsorbed fractions. LDL (the $d>1.019$ infranatant lipoprotein of the adsorbed fraction) (3) contained the major proportion ( $>90 \%$ ) of free and esterified cholesterol and phospholipids of VLDL + LDL in both fasting and postprandial plasma. Additionally, fasting $\mathrm{LDL}$ contained $49 \pm 10 \%$ of total triglyceride recovered and $29 \pm 8 \%$ of postprandial triglyceride in the VLDL + LDL fraction. As shown in Table II, lipemia was associated with significant decrease $(P$ $<0.001)$ in the ratio of free cholesterol and phospholipid in

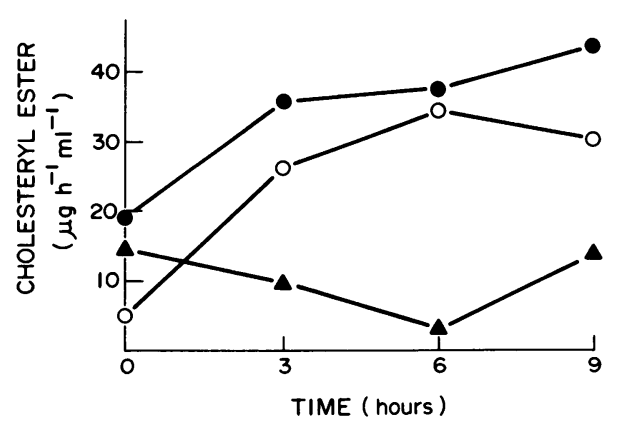

Figure 7. Time course of cholesteryl ester synthesis in plasma (- - -), cholesteryl ester transfer to VLDL + LDL (open circles), and cholesteryl ester accumulation in HDL ( $-\Delta-)$, determined as described in the legend to Fig. 6 . The values are those in fasting plasma and 3, 6, and $9 \mathrm{~h}$ postprandially. The data represent those obtained in four studies over this time course. 


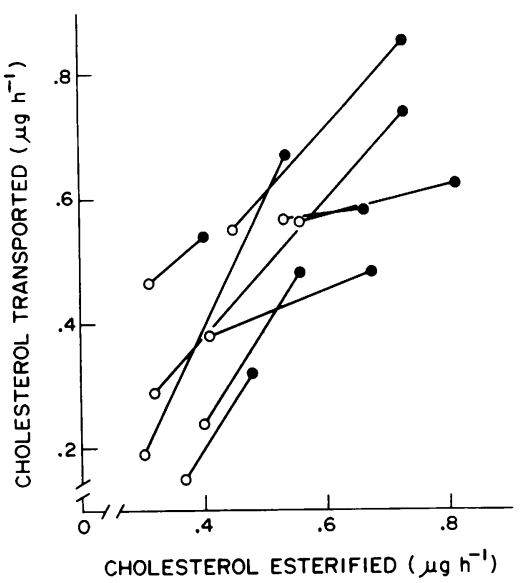

Figure 8. Relationship between total cholesterol esterified and cholesterol net transport from cell membranes for esterification, in fasting plasma $(-\circ-)$ and $5 \mathrm{~h}$ postprandially $(-\bullet-)$ in experiments where both were measured simultaneously in $1.2 \% \mathrm{vol} / \mathrm{vol}$ plasma in phosphate-buffered saline for $60 \mathrm{~min}$ at $37^{\circ} \mathrm{C}$. The values shown relate to transport and total free cholesterol esterification per dish of cells.

the LDL fraction. There was an overall decrease in LDL cholesteryl ester content, but the change in cholesteryl ester/ triglyceride ratio did not reach significance. The response of the two fractions of VLDL separated by heparin-agarose chromatography was quite distinct. In fasting plasma, $43 \pm 8 \%$ of total VLDL triglyceride recovered was not retained by heparin-agarose, but in postprandial plasma this proportion had decreased to $18 \pm 3 \%$, as total VLDL triglyceride increased 3.5 -fold. Accordingly, essentially the whole $(92 \pm 5 \%)$ of the total increase in VLDL triglyceride in postprandial plasma was in the fraction of VLDL adsorbed to heparin-agarose. A previous study (4) indicated that the adsorbed fraction of VLDL contained apolipoprotein $\mathrm{E}$, while the nonadsorbed contained no detectable apolipoprotein E. This finding was confirmed by isoelectric focusing for both fasting and postprandial VLDL. There was no difference in the pattern of Capolipoproteins of either adsorbed or nonadsorbed V.LDL

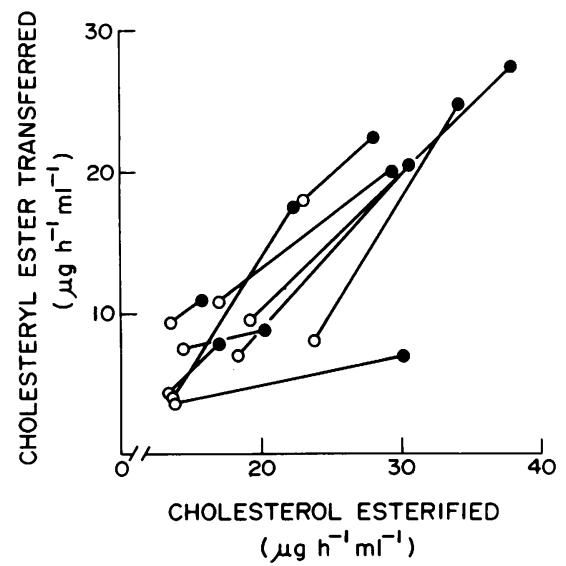

Figure 9. Relationship between cholesterol esterification and the rate of cholesteryl ester transfer to VLDL + LDL in fasting (-० -) and $5 \mathrm{~h}$ postprandial plasma $(-\bullet-)$. Determinations were made from the same plasma samples before and after incubation for $60 \mathrm{~min}$ at $37^{\circ} \mathrm{C}$.

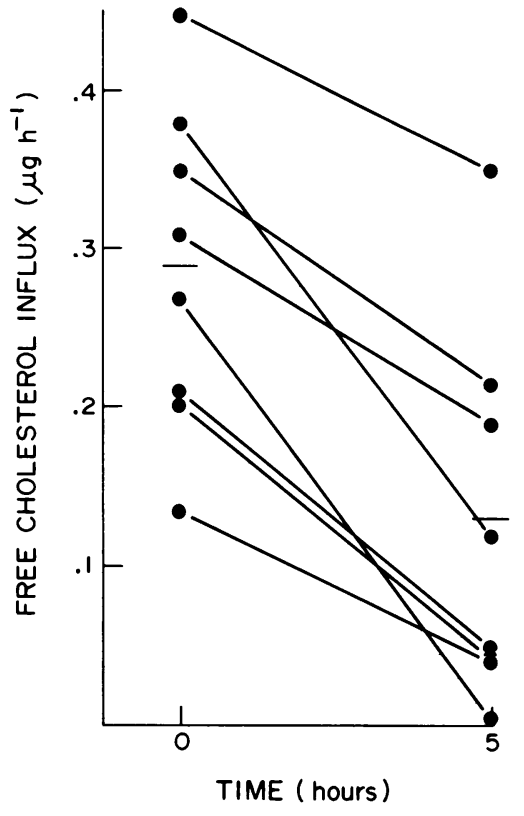

Figure 10. Influx of cholesterol from plasma lipoproteins to cell membranes. Influx was determined as efflux - net transport for cells as in the legend to Fig. 2 . The cells were prelabeled $(48 \mathrm{~h})$ with medium containing $1,2^{3} \mathrm{H}$-cholesterol, then washed and incubated with unlabeled plasma medium in phosphate-buffered saline. Efflux was determined from the rate of appearance of medium cholesterol radioactivity, and from the specific activity of cholesterol in unincubated cells labeled under the same conditions. Cholesterol net transport was determined from the rate of decrease in medium free cholesterol in empty dishes, and in dishes containing cells, as described in the legend to Fig. 2.

fractions of either fasting or postprandial plasma (data not shown), indicating that these polypeptides, unlike apo $\mathrm{E}$, readily equilibrate between VLDL species. In the same way, the major portion $(87 \pm 6 \%)$ of the increase in cholesteryl ester recovered in total VLDL was in the fraction adsorbed to heparin-agarose. These data support the earlier conclusion (4) that VLDL lacking app E plays little role as an acceptor for LCAT-derived cholesteryl esters.

\section{Discussion}

This study indicates that in postprandial lipemia there is a significant increase in the rate of net transport of free cholesterol from cell membranes to plasma, that is associated in turn with increases in the plasma rate of cholesterol esterification, and in the rate of transfer of the cholesteryl ester so formed to VLDL and LDL (1). Furthermore, although the lipemia followed the ingestion of a meal relatively rich $(0.6 \% \mathrm{wt} / \mathrm{wt})$ in cholesterol as dietary fat, there was actually a decrease in the proportion of cholesterol, both free and esterified, in the major acceptor lipoproteins (LDL) caused by the fact that although free cholesterol levels in plasma increased, the increases in triglyceride and phospholipid were much more substantial. This no doubt reflects the much higher proportion of glycerides than cholesterol in the diet used here, as in all normal diets.

It is a major conclusion of this study that postprandial lipemia, under these conditions, was associated with an increase in the transport of free cholesterol from cell membranes to plasma, in response to the increased demand for cholesterol associated with the activation of LCAT. Indeed, the major 
Table II. Lipid Composition of VLDL and LDL Fractions from Fasting and Postprandial Plasma

\begin{tabular}{|c|c|c|c|c|c|c|}
\hline & FC & PL & CE & TG & FC/PL & $\mathrm{CE} / \mathrm{TG}$ \\
\hline & weight percent & weight percent & weight percent & weight percent & & \\
\hline \multicolumn{7}{|l|}{ VLDL adsorbed } \\
\hline \multirow[t]{2}{*}{ fasting } & $7.3 \pm 1.5$ & $21.8 \pm 3.6$ & $13.8 \pm 3.4$ & $56.6 \pm 6.4$ & $0.34 \pm 0.07$ & $0.25 \pm 0.09$ \\
\hline & $P<0.001$ & $P<0.05$ & $P<0.001$ & $P<0.005$ & & $P<0.001$ \\
\hline postprandial & $5,0 \pm 2.0$ & $16.6 \pm 4.0$ & $8.3 \pm 3.3$ & $69.4 \pm 8.0$ & $0.30 \pm 0.07$ & $0.13 \pm 0.07$ \\
\hline \multicolumn{7}{|l|}{ VLDL nonads. } \\
\hline fasting & $3.6 \pm 0.6$ & $19.7 \pm 5.7$ & $3.5 \pm 0.7$ & $72.8 \pm 6.9$ & $0.19 \pm 0.04$ & $0.05 \pm 0.01$ \\
\hline postprandial & $3.3 \pm 0.6$ & $18.7 \pm 3.0$ & $3.9 \pm 1.3$ & $74.7 \pm 3.3$ & $0.19 \pm 0.03$ & $0.05 \pm 0.02$ \\
\hline \multicolumn{7}{|l|}{ LDL } \\
\hline \multirow[t]{2}{*}{ fasting } & $11.6 \pm 2.0$ & $27.8 \pm 4.0$ & $50.4 \pm 6.6$ & $9.9 \pm 1.7$ & $0.42 \pm 0.03$ & $5.32 \pm 1.70$ \\
\hline & & $P<0.02$ & $P<0.01$ & & $P<0.001$ & \\
\hline postprandial & $12.8 \pm 1.1$ & $33.3 \pm 3.2$ & $43.0 \pm 2.8$ & $11.1 \pm 1.8$ & $0.38 \pm 0.03$ & $4.10 \pm 0.88$ \\
\hline
\end{tabular}

Values represent the means \pm SD of eight experiments. Phospholipid (PL) mass was calculated on the basis of MW 750 and cholesteryl ester (CE) as cholesteryl oleate. Triglyceride was calculated as tripalmitin. $P$ values are determined on the basis of the difference in composition of fasting and postprandial lipoproteins of individual donors. $P$ values of $>0.05$ were considered not significant.

part of the extra free cholesterol required was supplied by the cells, even in the presence of a slightly increased concentration of medium plasma lipoprotein cholesterol. Furthermore, this was shown in each case to be the result of a decrease in the rate of influx of free cholesterol from plasma lipoproteins to the cells, in the face of a basically unchanged efflux rate. The overall effect of these changes is that LCAT uses cell cholesterol even more efficiently (in relation to plasma lipoprotein cholesterol) in postprandial lipemia than it does during fasting. It has been previously shown (4) that when VLDL and LDL contain an increased proportion of cholesterol, relative to phospholipid, there is a chemical potential gradient of free cholesterol established between these lipoproteins and the cell membranes, which drives an increased influx of cholesterol into the cells. Indeed, under these conditions, the concentration of free cholesterol in the lipoprotein surface may reach saturation in terms of the phase diagram of lipoprotein lipids (4). The finding in the present study that a decreased free cholesterol (FC)/phospholipid (PL) ratio in VLDL and LDL overall is associated with a decreased rate of influx into cell membranes in postprandial lipemia, suggests that a similar mechanism, but in the opposite sense, may be operative here. The following evidence supports this concept. First, the relationship between changes in FC/PL ratio and influx is similar in both cases. In diabetes, a change in transport of $-0.4 \mu \mathrm{g}$ cholesterol $\mathrm{h}^{-1}$ was associated with an increase in FC/PL ratio $0.10(3,4)$. In the present study, an increase in cholesterol transport out of the cells of $+0.19 \mu \mathrm{g} \mathrm{h}^{-1}$ was associated with a decrease in VLDL and LDL FC/PL ratio of 0.04 . This comparison supports the concept of a linear relationship between cholesterol transport and FC/PL ratio over the physiological range. Secondly, just as the increased FC/PL ratio of VLDL and LDL in diabetics is associated with a greatly reduced rate of cholesteryl ester transfer to VLDL and LDL, so the decreased FC/PL ratio of postprandial plasma is associated with an increase in transfer to the same lipoprotein classes. In the former case a decrease in transfer of $12.5 \mu \mathrm{g}$ cholesteryl ester $\mathrm{ml}^{-1}$ plasma $\mathrm{h}^{-1}$ was associated with a change in FC/PL ratio $( \pm 0.10)$ in VLDL and LDL (4). In the present experiments, a decrease in FC/PL ratio postprandially of 0.04 in $\mathrm{LDL}$ and the adsorbed fraction of VLDL, the major recipients of transferred cholesteryl ester (4), was associated with an increase in transfer rate of $+7.0 \mu \mathrm{g}$ cholesteryl ester $\mathrm{ml}^{-1}$ plasma $\mathrm{h}^{-1}$. These findings seem to argue strongly that the basic mechanism of the effects of FC/PL ratio is the same in lipemia and in diabetes. These data seem to argue strongly that the decreased influx of free cholesterol from the plasma, and hence the improved net transport of cholesterol from cell membranes to the LCAT reaction, is the direct result of the decreased FC/PL ratio of VLDL and LDL in postprandial plasma. The significance of even small changes in this ratio for the phase distributions of lipoprotein surface lipids has been previously stressed (15). A separate study (16) of lipoprotein composition in human hypertriglyceridemia of genetic origin indicated that there was a decrease in cholesterol saturation in LDL. If these subjects were similar to the group of hypertriglyceridemic subjects studied in our own laboratory (2), who showed cholesterol transport and transfer rates in excess of those found in normolipidemic controls, these data provide an independent model for the effect of FC/PL ratio on plasma cholesterol metabolism.

The second finding was that the rate of esterification of cholesterol in plasma was stimulated in postprandial lipemia. This confirms an earlier report (17) where the stimulation $(\sim 1.7$-fold) reported was similar to that found in the present study. Here also, the isolated postprandial lipoproteins stimulated cholesterol esterification when added to fasting plasma. A smaller increment of esterification $(\sim 1.3$-fold) was found in a second study (18). As discussed above, the present increase in esterification is well-correlated with the increase in cholesterol net transport. It is unlikely that the increased rate is the direct result of the mass of cellular cholesterol used for esterification. Earlier, direct comparison of LCAT rates with lipoproteinderived and membrane-derived cholesterol failed to demonstrate any difference in esterification rates (7). Additionally, there was no difference in the absolute mass of LCAT in plasma in fasting and postprandial plasma, as determined from esterification rate with a liposome substrate under conditions independent of plasma lipoprotein concentrations. Finally, it is 
unlikely that changes in the lipoprotein donor of free cholesterol for LCAT between HDL and VLDL + LDL mediates this increase in activity. There is only a small change in the proportion of free cholesterol supplied by HDL in the two conditions, much less numerically than the increase in the rate of esterification overall. Accordingly, the increase in esterification is most likely to result from a release of inhibition mediated by the lipoprotein recipient of the LCAT-derived cholesteryl ester. Since the major amount $(\sim 90 \%)$ of this increased rate of transfer is directed to the VLDL and LDL fractions of plasma, it must be changes in the properties of these lipoproteins seen in postprandial plasma that mediate the increased rate of esterification seen. This concept receives support from the finding that the increments obtained in unfractionated postprandial plasma in cholesterol net transport, esterification, and transfer are comparable with the changes observed when VLDL + LDL from such plasma are removed and added to preprandial plasma.

Two main changes were seen in the lipid composition of these lipoproteins. First, in LDL there was a significant decrease in the FC/PL ratio. Second, in the adsorbed fraction of VLDL, and to a lesser extent in LDL, there was an increase in the ratio of triglyceride to cholesteryl ester. While these changes cannot be correlated directly with in vitro transfer rates (because of changes in lipoprotein secretion and clearance in vivo over the 5-h postprandial interval), nevertheless, they throw light on mechanisms and transfer in postprandial lipemia. On the basis of recent models of the cholesteryl ester transfer reaction (19), it would be predicted that since the reaction involves in plasma an exchange of cholesteryl ester for triglyceride $(20,21)$, the triglyceride/cholesteryl ester ratio of the lipoprotein acceptor would be the primary determinant of reaction rate. However, several considerations indicate that this is not the only, and may not be the most important, factor in postprandial plasma. First, at least in fasting plasma incubated in vitro, the major recipient of LCAT-derived cholesteryl ester is LDL, even though the greatest change in composition is seen in VLDL (22). Since LDL triglyceride content is, if anything, increased, it seems likely that LDL is also a major acceptor of cholesteryl ester in postprandial plasma. Second, the transfer rate in native plasma is linear for considerable periods during which VLDL and LDL triglyceride is exchanged for cholesteryl ester (22). This indicates that the triglyceride/cholesteryl ester ratio is not rate limiting at the ratios found in fasting plasma, and could therefore hardly be so at the higher ratios present in postprandial lipoproteins. Third, as discussed above, the data obtained for the relationship between FC/PL ratio and transfer from postprandial plasma are consistent with that obtained earlier (4) for diabetic plasma, yet in the latter case, a decrease in transfer was associated with an increased or unchanged triglyceride (TG)/cholesteryl ester (CE) ratio, probably mediated by the decrease in transfer rate per se. Finally, the proportion of VLDL + LDL triglyceride in LDL decreases only from $50 \%$ in fasting plasma to $30 \%$ in postprandial plasma, while the maximum rate of transfer to VLDL only exceeded that to LDL by about $15 \%$ (21). These changes seem much too small to explain a $100 \%$ increase in the rate of cholesteryl ester transfer to VLDL and LDL. While a contribution by $\mathrm{TG} / \mathrm{CE}$ ratio in determining transfer in lipemia is by no means excluded by the present data, the evidence here suggests that (as in diabetic plasma) the FC/PL ratio may contribute importantly to determine transfer rate.
Whether this effect involves a change in the physical properties of the lipoprotein surface or some other factor (affecting, for example, the conformation of the transfer protein itself at the surface) cannot at present be determined. The protein composition of the VLDL particles is unlikely to be a factor, since transfer occurs at good rates to synthetic acceptor surfaces containing no apoproteins at all (23). The finding that the increase of VLDL triglyceride was limited to particles retained by heparin-agarose no doubt reflects the earlier finding (2425) that in lipemia, apo E moves from HDL into VLDL and LDL. However, there seems to be no evidence to this point that apo $\mathrm{E}$ is required for cholesteryl ester transfer, even though apo $\mathrm{E}$ may passively redistribute to VLDL rich in cholesteryl esters.

In summary, this research provides further evidence for the integration of cholesterol net transport, esterification, and transfer rates, as shown by the correlations obtained between stimulated and unstimulated rates in fasting and postprandial lipemia. It also suggests, in wider terms, that postprandial lipemia may play an important role in stimulating the return of peripheral cell cholesterol to hepatic degradation sites by the mechanism described here. This stimulus is still significant, in normal subjects, after a fat meal containing physiological levels of dietary cholesterol.

\section{Acknowledgments}

This research was supported by Arteriosclerosis SCOR HL-14237 and by HL-23738 of the National Heart, Lung and Blood Institutes. The expert technical assistance of Mary Fisher and Elaine Hoye is acknowledged.

\section{References}

1. Fielding, C. J., and P. E. Fielding. 1982. Cholesterol transport between cells and body fluids. Role of plasma lipoproteins and the plasma cholesterol esterification system. Med. Clin. N. Am. 66:363373.

2. Fielding, P. E., C. J. Fielding, R. J. Havel, J. P. Kane, and P. Tun. 1983. Cholesterol net transport, esterification, and transfer in human hyperlipidemic plasma. J. Clin. Invest. 71:449-460.

3. Fielding, C. J., G. M. Reaven, and P. E. Fielding. 1982. Human noninsulin-dependent diabetes: identification of a defect in plasma cholesterol transport normalized in vivo by insulin and in vitro by selective immunoadsorption of apolipoprotein E. Proc. Natl. Acad. Sci. USA. 79:6365-6369.

4. Fielding, C. J., G. M. Reaven, G. Liu, and P. E. Fielding. 1984. Increased free cholesterol in plasma low and very low density lipoproteins in noninsulin-dependent diabetes mellitus: its role in the inhibition of cholesteryl ester transfer. Proc. Natl. Acad. Sci. USA. 81:2512-2516.

5. Kuksis, A., J. J. Myher, K. Geher, G. J. L. Jones, W. C. Breckenridge, T. Feather, D. Hewitt, and J. A. Little. 1982. Decreased plasma phosphatidyl choline/free cholesterol ratio as an indicator of risk for ischemic vascular disease. Arteriosclerosis. 2:296-302.

6. Fielding, P. E., P. M. Davison, M. A. Karasek, and C. J. Fielding. 1982. Regulation of sterol transport in human microvascular endothelial cells. J. Cell Biol. 94:350-354.

7. Fielding, C. J., and P. E. Fielding. 1981. Evidence for a lipoprotein carrier in human plasma catalyzing sterol efflux from cultured fibroblasts and its relationship to lecithin: cholesterol acyltransferase. Proc. Natl. Acad. Sci. USA. 78:3911-3914.

8. Fielding, C. J. 1984. Lecithin: cholesterol acyltransferase and cholesterol transport. In Methods in Enzymology: Steroids. J. H. Law and H. Rilling, editors. Academic Press Inc., New York. 111:267-274.

9. Calison, L. A. 1963. Determination of serum triglycerides. J. Atheroscler. Res. 3:334-336. 
10. Bartlett, G. R. 1959. Phosphorus assay in column chromatography. J. Biol. Chem. 234:466-468.

11. Pagnan, A., R. J. Havel, J. P. Kane, and L. Kotite. 1977. Characterization of human very low density lipoproteins containing two electrophoretic populations: double pre-beta lipoproteinemia and dysbetalipoproteinemia. J. Lipid Res. 18:613-622.

12. Castro, G. R., and C. J. Fielding. 1984. Evidence for the distribution of apolipoprotein $\mathrm{E}$ between lipoprotein classes in human normocholesterolemic plasma and for the origin of unassociated apoprotein E (Lp-E). J. Lipid Res. 25:58-67.

13. Lowry, O. H., N. J. Rosebrough, A. L. Farr, and R. J. Randall. 1951. Protein measurement with the Folin phenol reagent. J. Biol. Chem. 193:265-275.

14. Aron, L., S. Jones, and C. J. Fielding. 1978. Human lecithin: cholesterol acyltransferase. Characterization of cofactor-dependent phospholipase activity. J. Biol. Chem. 253:7220-7226.

15. Miller, K. W., and D. M. Small. 1983. Surface-to-core and interparticle equilibrium distributions of triglyceride-rich lipoprotein lipids. J. Biol. Chem. 258:13772-13784.

16. Vakakis, N., T. G. Redgrave, D. M. Small, and W. P. Castelli. 1983. Cholesterol content of red blood cells and low density lipoproteins in hypertriglyceridemia. Biochim. Biophys. Acta. 751:280-285.

17. Marcel, Y. L., and C. Vezina. 1973. Lecithin:cholesterol acyltransferase of human plasma. Role of chylomicrons, very low and high density lipoproteins in the reaction. J. Biol. Chem. 248:82548259.
18. Rose, H. G., and J. Juliano. 1977. Regulation of plasma lecithin:cholesterol acyltransferase. Activation during alimentary lipemia. J. Lab. Clin. Med. 89:524-532.

19. Ihm, J., D. M. Quinn, S. J. Busch, B. Chataing, and J. A. K. Harmony. 1982. Kinetics of plasma protein-catalyzed exchange of phosphatidylcholine and cholesteryl ester between plasma lipoproteins. J. Lipid Res. 23:1328-1341.

20. Rehnborg, C. S., and A. V. Nichols. 1964. The fate of cholesteryl esters in human serum incubated in vitro at $38^{\circ}$. Biochim. Biophys. Acta. 84:596-603.

21. Chajek, T., and C. J. Fielding. 1978. Isolation and characterization of a human serum cholesteryl ester transfer protein. Proc. Natl. Acad. Sci. USA. 75:3445-3449.

22. Fielding, C. J., and P. E. Fielding. 1981. Regulation of human plasma lecithin:cholesterol acyltransferase activity by lipoprotein acceptor cholesteryl ester content. J. Biol. Chem. 256:2102-2104.

23. Chajek, T., L. Aron, and C. J. Fielding. 1980. Interaction of lecithin:cholesterol acyltransferase and cholesteryl ester transfer protein in the transport of cholesteryl ester into sphingomyelin liposomes. Biochemistry. 19:3673-3677.

24. Vigne, J.-L., and R. J. Havel. 1981. Metabolism of apolipoprotein A-I of chylomicrons in rats and humans. Can. J. Biochem. 59:613618.

25. Blum, C. B. 1982. Dynamics of apolipoprotein E metabolism in humans. J. Lipid Res. 23:1308-1316. 